\title{
Abordagem multiprofissional na pneumonia associada à ventilação mecânica
}

\author{
Multiprofessional approach in pneumonia associated with \\ mechanical ventilation
}

\section{Enfoque en neumonía multiprofesional asociada a la ventilación mecánica}

Marcos Vinícius da Conceição Furtado ${ }^{1 *}$, Jamile Corrêa Silva ${ }^{1}$, Augusto Cezar Ferraz da Costa', Letícia Maues Marques ${ }^{1}$, Victória Fernanda Barbosa ${ }^{1}$, Jhensela Silva dos Prazeres ${ }^{1}$, Ramon Moraes de Moraes ${ }^{1}$, Cláudio Alves do Amaral'1.

\section{RESUMO}

Objetivo: Apresentar diferentes estudos que abordem sobre os fatores de riscos, prevenção e tratamento da pneumonia associada à ventilação mecânica na unidade de terapia intensiva, para assim informar e nortear as condutas da equipe multiprofissional de saúde diante desta afecção. Métodos: O presente artigo trata-se de uma revisão sistemática fundamentada em pesquisas realizadas nos indexadores de dados eletrônicos PubMed, Scielo e Pedro, onde foram feitas análises críticas e minuciosas dos estudos, com a justificativa de extrair as evidências de alta qualidade. Resultados: Evidenciou-se que a pneumonia associada a ventilação mecânica aumenta a taxa de mortalidade e o tempo de permanência em ventilação mecânica, com isso reuniu-se dez estudos em uma tabela com o objetivo de apresentar os fatores de riscos que potencializam o surgimento desta infecção, as condutas para a prevenção e os protocolos para o tratamento. Considerações finais: Podemos concluir que a pneumonia associada à ventilação mecânica pode ter sua incidência reduzida nas unidades de terapia intensiva realizando certas medidas como higienização bucal, fisioterapia respiratória, administração de probióticos, drenagem de secreções e monitorização adequada do paciente. Contudo, verificou-se que são poucos os estudos que demonstram os impactos das condutas em outros desfechos clínicos.

Palavras-chave: Infecções respiratórias, Pneumonia associada a ventilação mecânica, Unidades de terapia intensiva.

\begin{abstract}
Objective: To present different studies that address the risk factors, prevention and treatment of pneumonia associated with mechanical ventilation in the intensive care unit, in order to inform and guide the conduct of the multidisciplinary health team in the face of this condition. Methods: This article is a systematic review based on research conducted in the electronic data indexers PubMed, Scielo and Pedro, where critical and thorough analyzes of the studies were carried out, with the justification of extracting high-quality evidence.. Results: It was evidenced that pneumonia associated with mechanical ventilation increases the mortality rate and the time spent on mechanical ventilation, thus bringing together ten studies in a table in order to present the risk factors that enhance the appearance of this infection, conducts for prevention and protocols for treatment. Final considerations: We can concluede that pneumonia associated with mechanical ventilation may have its incidence reduced in intensive care units by performaing certain measures such as oral hygiene, respiratory physiotherapy, administration of probiotics, drainage of secretions and adequate monitoring of the patient. However, it was found that there are few studies that demonstrate the impacts of conducts on other clinical outcomes.
\end{abstract}

Keywords: Respiratory tract infections, Pneumonia Ventilador-associated, Intensive care units

\footnotetext{
${ }^{1}$ Escola Superior da Amazônia (ESAMAZ), Belém-PA. *E-mail: viniifurtado97@gmail.com
}

SUBMETIDO EM: 6/2020 ｜ ACEITO EM: 7/2020 ｜ＰUBLICADO EM: 10/2020




\section{RESUMEN}

Objetivo: Presentar distintos estudios que aborden los factores de riesgo, la prevención y el tratamiento de la neumonía asociada a la ventilación mecánica en la unidad de cuidados intensivos, con el fin de informar y guiar la conducta del equipo de salud multidisciplinario ante esta condició. Métodos: Este artículo es una revisión sistemática basada en una investigación realizada en los indexadores electrónicos de datos PubMed, Scielo y Pedro, donde se llevaron a cabo análisis críticos y estudios exhaustivos, con la justificación de extraer evidencia de alta calidad. Resultados: Se evidenció que la neumonía asociada con la ventilación mecánica aumenta la tasa de mortalidad y el tiempo dedicado a la ventilación mecánica, reuniendo así diez estudios en una tabla para presentar los factores de riesgo que mejoran la apariencia de este infección, conductas de prevención y protocolos de tratamiento. Consideraciones finales: Podemos concluir que la neumonía asociada con la ventilación mecánica puede reducir su incidencia en las unidades de cuidados intensivos mediante la realización de ciertas medidas, como la hygiene oral, la fisioterapia respiratoria, la administración de probióticos, el drenaje de secreciones y la monitorización adecuada del paciente. Sin embargo, se encontró que hay pocos studios que demuestren los impactos de las condutas en otros resultados clínicos.

Palabras clave: Infecciones del sistema respiratorio, Neumonía asociada al ventilador, Unidades de cuidados intensivos.

\section{INTRODUÇÃO}

As práticas assistenciais de qualidade e seguras são um desafio para os profissionais e instituições de saúde, não se configurando como um dogma recente. Percebe-se que são escassas as intervenções efetivas e de qualidade que proporcionam uma assistência humanizada e técnica. A busca constante da excelência no entendimento das diversas patologias que compõe e moldam o cotidiano hospitalar se torna crucial, devendo os profissionais que compõe a equipe multiprofissional buscar constantemente por atualizações técnicas (BARROS F, et al., 2019).

Dentro do contexto hospitalar destacamos a Unidade de Terapia Intensiva (UTI), um ambiente planejado com o objetivo de agrupar pacientes instáveis e recuperáveis, tecnologias de ponta e recursos humanos capacitados, exigindo agilidade e atendimento qualificado e específico. Porém, por mais que pacientes admitidos nessas unidades se beneficiem da sua multiprofissionalidade assistencial e tecnológica, eles têm de 5 a 10 vezes mais probabilidade de desenvolver Infecções Relacionadas à Assistência à Saúde (IRAS), principalmente Pneumonia Associada à Ventilação Mecânica (PAV), devido ao elevado número de procedimentos e dispositivos invasivos que prolongam o tempo de estadia nesse ambiente (HESPANHOL L, et al., 2019; BARROS F, et al., 2019).

A ventilação mecânica (VM) serve como um método de suporte para o paciente durante uma enfermidade aguda, sendo utilizada até a sua resolução. O emprego da ventilação mecânica(VM) implica riscos próprios, devendo sua indicação ser prudente e criteriosa, devendo sua aplicação ser cercada por cuidados específicos. As principais indicações para inserir o individuo em ventilação mecânica (VM) são nas situações de emergência, tais como parada cardiorrespiratória, insuficiência respiratória, hipoventilação, choque, coma, pós-operatório e politraumatismo. Para minimizar os riscos, o médico deve realizar a avaliação inicial do paciente com relação a seu nível de consciência, fatores de risco para aspiração pulmonar e presença de via aérea difícil (YAMANAKA CS, et al., 2010; BARBAS CSV, et al., 2014).

Sabe-se que com o passar dos dias em cuidados intensivos e fazendo uso de prótese ventilatória invasiva, ou seja, estando sob ventilação mecânica (VM), surge no paciente crítico uma espécie de placa dentária que se configura como um biofilme complexo que, uma vez formado é relativamente resistente ao controle químico. Os agentes microbianos se acumulam rapidamente na boca do paciente sob suporte ventilatório invasivo e, à medida que a quantidade de placa aumenta, é provável o aumento da colonização por patógenos microbianos. A colonização da placa pode ser exacerbada na ausência de higienização bucal e pela secagem da cavidade oral devido à abertura prolongada da boca, o que reduz os efeitos de tamponamento e limpeza 
oral através da saliva, acarretando em um grande fator para o surgimento de pneumonia associada a ventilação mecânica (PAV) (HUA F, et al., 2016; ROQUILLY A, et al., 2015).

A pneumonia associada a ventilação mecânica (PAV) é um subconjunto de pneumonia nosocomial, que ocorre 48 horas ou mais após a inserção do paciente em ventilação mecânica (VM) via tubo endotraqueal ou de traqueostomia, sendo os mais importantes dos microrganismos responsáveis pela infecção as bactérias Staphylococcus aureus ( $S$. aureus), Pseudomonas aeruginosa ( $P$. Aeruginosa), e Enterobacteriaceae. Entretanto, os agentes etiológicos diferem amplamente de acordo com a população de pacientes em uma unidade de terapia intensiva (UTI). A pneumonia associada a ventilação mecânica (PAV) pode ser classificada como precoce quando ocorre até o quarto dia de intubação ou tardia quando ocorre após esse período. Em geral, a pneumonia associada a ventilação mecânica (PAV) inicial é causada por microrganismos sensíveis a antibióticos, enquanto a de início tardio é causada por patógenos resistentes a vários medicamentos, sendo o tratamento mais difícil (ANVISA 2017; RODRIGUES AN, et al., 2016; CHOUHDARI A, et al., 2018).

A Agencia Nacional de Vigilância Sanitária(ANVISA), relata que os fatores que podem potencializar o surgimento da pneumonia associada a ventilação mecânica (PAV) são multivariados, como: idade avançada (acima de 70 anos); coma; nível de consciência; intubação e reintubação traqueal; condições imunitárias; uso de drogas imunodepressoras; choque; gravidade da doença; antecedência de doença pulmonar obstrutiva crônica (DPOC); tempo prolongado de ventilação mecânica (VM) maior que sete dias; aspirado do condensado contaminado dos circuitos do ventilador; desnutrição; antibioticoterapia como profilaxia e cirurgias prolongadas (ANVISA, 2017).

A incidência de pneumonia hospitalar em pacientes intubados é 7 a 21 vezes maior do que em não intubados, sendo responsável por $15 \%$ das Infecções Relacionadas a Assistencia a Saúde (IRAS), tendo o percentual aproximadamente de $25 \%$ de todas as infecções adquiridas nas unidades de terapia intensiva (UTI), onde o tempo de permanência pode aumentar em 6,1 dias com um custo médio de US\$382,00 a US\$ 1.833,00 dólares adicionais, repercutindo de maneira significativamente negativa nos desfechos dos individuos(BARROS F, et al., 2019). Outro estudo conduzido em 99 hospitais brasileiros, verificou que a pneumonia associada a ventilação mecânica (PAV) foi responsável por $28,9 \%$ de todas as infecções nosocomiais e, destas, $50 \%$ ocorreram em pacientes ventilados mecanicamente. Além disso, constatou-se que o índice de mortalidade atribuída a essa infecção é diferente nos diversos estudos, no entanto, evidenciou-se que aproximadamente $33 \%$ dos pacientes vão a óbito em decorrência direta desta, traduzindose no prolongamento da hospitalização por volta de 12 dias e no aumento de custos hospitalares em torno de 40.000 mil dólares por episódio (RODRIGUES AN, et al., 2016; CARVALHO CRR, 2006).

Embora exista várias definições de pneumonia associada a ventilação mecânica (PAV) e tempo de início de sua instalação, é notável alguns achados clínicos e radiológicos encontrados nos pacientes sob ventilação mecânica (VM) que possibilitam o diagnóstico preciso, sendo alguns deles: febre acima de $38^{\circ} \mathrm{C}$, secreção purulenta no trato respiratório, radiografia de tórax com presença de infiltrados pulmonares progressivos e cultura microbiológica. Esses achados servem como medidas para diagnosticar a pneumonia associada a ventilação mecânica (PAV), sendo os exames de baixos custos, o que possibilita o seu uso na unidade de terapia intensiva (UTI) (LIU W, et al., 2019; FERREIRA CR, et al., 2016).

Além disso, a alta incidência e mortalidade atribuida à esta infecção está associada ao aumento da carga de utilização de recursos humanos, assim sendo necessárias intervenções custo-efetivas que minimizem o surgimento desse evento adverso. Todavia, se nota o grande desafio para a implementação de protocolos preventivos contra a pneumonia associada a ventilação mecânica (PAV) nos centros de terapia intensiva, uma vez que esses protocolos buscam modificar o comportamento dos profissionais de saúde. No entanto, pode haver disseminação de boas práticas ao serem adotadas as técnicas corretas por meio de uma abordagem participativa de todos os profissionais da equipe multiprofissional de saúde (CARRASCOSA DP, et al., 2020; ANVISA, 2017).

A implementação de pacotes preventivos e de de medidas curativas baseadas em evidências científicas se tornou um método de sucesso na prática clínica. Sua eficácia se justifica no agrupamento das melhores práticas assistenciais que, quando usadas individualmente, podem não ter o desfecho desejado, porém, tal 
sucesso depende do envolvimento da equipe multiprofissional, onde suas ações devem ser centradas na seleção das intervenções, conforme as necessidades de cada unidade e individuo, havendo com frequencia treinamentos para a assistência e fiscalização da adesão diante dos profissionais (BARROS F, et al., 2019).

Paralelo a isso, evidenciou-se que algumas das condutas utilizadas por membros que compõem a equipe multiprofissional, são capazes de promover a prevenção e o tratamento da pneumonia associada a ventilação mecânica (PAV), tais como o uso de posições corretas no leito, cuidados de higienização bucal, antibióticoterapia, técnicas de fisioterapia respiratória, correta alimentação e monitorização devida dos cuidados oferecidos aos pacientes (HUA F, et al., 2016; FERREIRA CR, et al., 2016; ALEIXOU VG, et al., 2009).

Diante do cenário, este artigo tem como objetivo realizar uma síntese dos diferentes estudos existentes na literatura, com a proposta de mostrar o impacto da pneumonia associada à ventilação mecânica(PAV) e, consequentemente, incentivar a prática de medidas de prevenção e formas de tratamentos mais eficazes por meio das equipes multiprofissionais das unidades de terapia intensiva (UTI's), norteando suas condutas conforme evidências relevantes encontradas e destacadas aqui.

\section{MÉTODOS}

Utilizou-se como fundamento para a realização desta revisão integrativa buscas de dados nas bases eletrônicas PubMed (US national Library of Medicine), Scielo (Scientific Electronic Library Online), Pedro (Physiotherapy Evidence Database) e materiais da Agência Nacional de Vigilância Sanitária (ANVISA).

Os anos de publicação dos materiais utilizados variaram de 2005 a 2020, sendo os idiomas português, inglês e espanhol dos estudos selecionados. Nas buscas foram utilizadas as seguintes palavras-chaves: Infecções Respiratórias; Pneumonia Associada a Ventilação Mecânica; Unidades de Terapia Intensiva.

Os critérios de inclusão definidos foram: 1) Pacientes submetidos a intubação orotraqueal ou traqueostomia, 2) Pacientes com o diagnóstico de pneumonia associada à ventilação mecânica, 3) Estudos que descrevam os fatores que potencializam o surgimento da pneumonia associada a ventilação mecânica, 4) Estudos com medidas de prevenção e protocolos de tratamento, 5) Pacientes com idade superior a 18 anos e 6) Pacientes de ambos os sexos. Os critérios de exclusão definidos foram: 1) estudos com outras infecções hospitalares, 2) estudos com dados incompletos e 3) estudos com público pediátrico ou neonatal.

No total foram encontrados 101 artigos, destes, 91 foram excluídos, alguns por abordarem diferentes tipos de infecções hospitalares, outros por analisarem o público neonatal/pediátrico, e também por apresentarem dados incompletos sobre os pacientes. Os 10 artigos restantes obedeceram aos critérios estabelecidos para inclusão, ocasionando assim na seleção dos mesmos.

Para elegibilidade dos estudos foram realizadas leituras das metodologias e conclusões/considerações finais. Os estudos com relação completa com a linha de pesquisa proposta foram lidos na íntegra de forma crítica, com a justificativa de incluir somente os estudos de alta qualidade.

Após seleção, os estudos foram encaminhados para a tabela, que apresenta as características dos estudos e seus principais desfechos. As buscas pelos artigos foram adaptada seguindo o modelo de fluxograma PRISMA 2009 FLOW DIAGRAM que nos indica por etapas as formas das buscas, bem como apresenta os critérios de seleção (Figura 1).

\section{RESULTADOS}

Os resultados dos dez estudos selecionados, juntamente com suas características e seus principais desfechos estão expostos na Tabela 1. 
Figura 1 - Fluxograma das buscas e seleção dos artigos conforme sugerido por PRISMA.

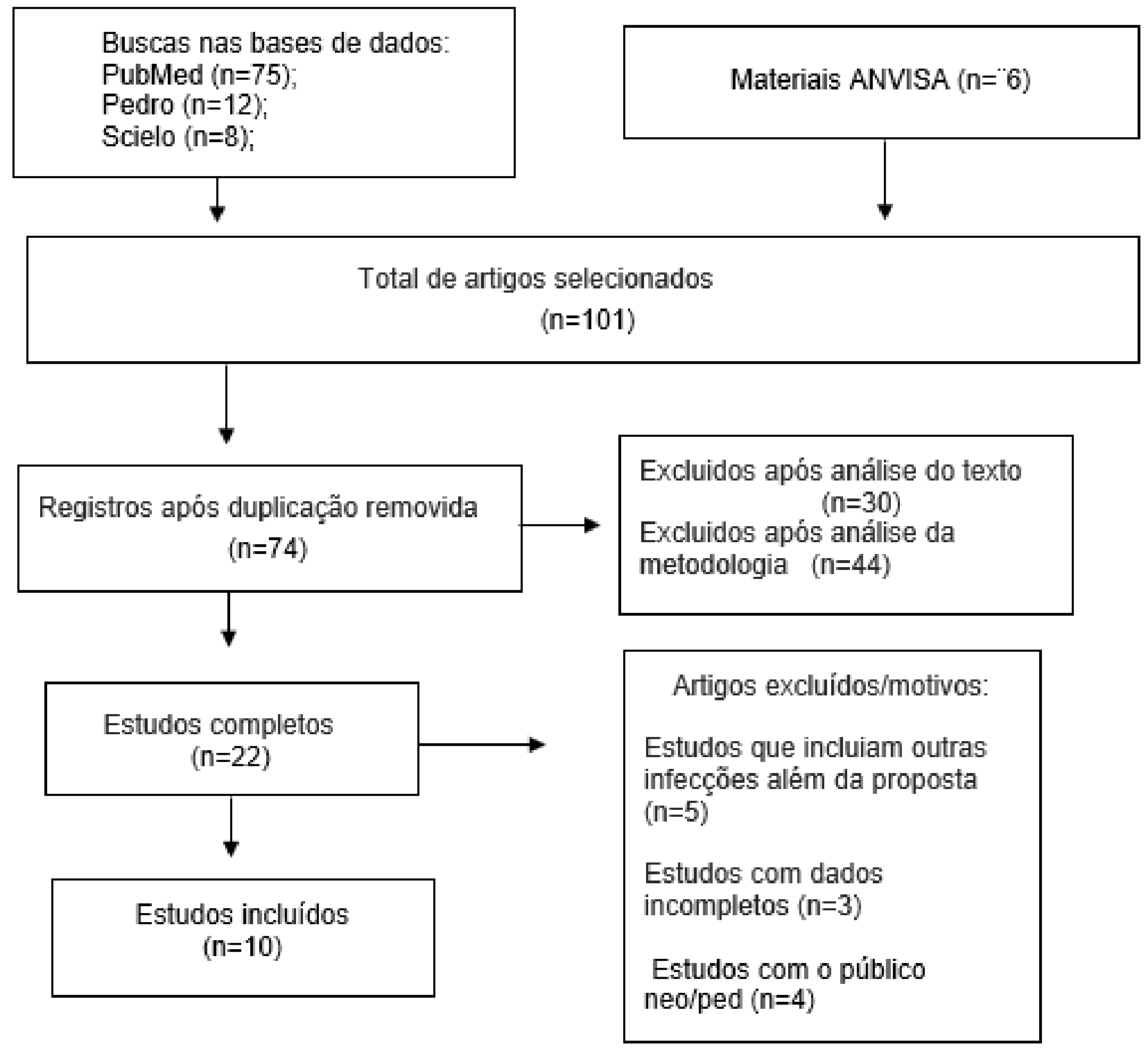

Fonte: Furtado MVC, et al., 2020. 
Revista Eletrônica Acervo Saúde / Electronic Journal Collection Health ｜ ISSN 2178-2091

Tabela 1 - Apresentação dos estudos selecionados com suas características e principais desfechos.

\begin{tabular}{|c|c|c|c|c|}
\hline Autor/ano & Título & $\begin{array}{l}\text { Amostra/ } \\
\text { Método }\end{array}$ & $\begin{array}{l}\text { Protocolo/ } \\
\text { Principais resultados das análises }\end{array}$ & Conclusão \\
\hline $\begin{array}{l}\text { HUA F, et al., } \\
2016\end{array}$ & $\begin{array}{l}\text { Cuidados de higiene bucal em } \\
\text { pacientes críticos para } \\
\text { prevenir pneumonia } \\
\text { associada ao ventilador }\end{array}$ & $\begin{array}{l}\text { Foram } \\
\text { incluídos } 38 \\
\text { ensaios } \\
\text { clínicos } \\
\text { randomizados } \\
\text { (6016 } \\
\text { participantes) }\end{array}$ & $\begin{array}{l}\text { Evidências de alta qualidade mostraram que o } \\
\text { enxaguatório bucal ou gel clorexidina, reduzem } \\
\text { o risco de PAV em comparação com o placebo } \\
\text { ou cuidados usuais, de } 24 \% \text { para cerca de } 18 \% \text {. }\end{array}$ & $\begin{array}{c}\text { Enxaguatório bucal com clorexidina ou gel, reduz o } \\
\text { risco de desenvolver pneumonia associada à } \\
\text { ventilação mecânica em pacientes críticos. No } \\
\text { entanto, não há evidências que mostrem diminuição } \\
\text { nos resultados de mortalidade, duração da } \\
\text { ventilação mecânica ou tempo de permanência na } \\
\text { UTI. }\end{array}$ \\
\hline $\begin{array}{l}\text { LIU W, et al., } \\
2019\end{array}$ & $\begin{array}{l}\text { Vigilância ativa da pneumonia } \\
\text { associada à ventilação } \\
\text { mecânica na unidade de } \\
\text { terapia intensiva e } \\
\text { estabelecimento do sistema de } \\
\text { classificação de risco e } \\
\text { avaliação de efeito. }\end{array}$ & $\begin{array}{l}\text { Um total de } \\
1.513 \\
\text { pacientes } \\
\text { analisados em } \\
\text { seis UTIs. }\end{array}$ & $\begin{array}{l}\text { Os indicadores que potencializaram a incidência } \\
\text { de PAV foram: Tempo de internação na UTI, } \\
\text { frequência de cuidados orais, drenagem de } \\
\text { secreção subglótica não realizada, } \\
\text { traqueostomia, escore APACHE II e uso } \\
\text { combinado de antibióticos }(\mathrm{P}<0,05) \text {. }\end{array}$ & $\begin{array}{l}\text { Após análises dos indicadores obtidos foram } \\
\text { aplicados os cuidados, e observou-se que a taxa de } \\
\text { incidência de PAV foi menor, com o percentual de } \\
\text { 13,22\% ( } 80 / 605) \text {. O que significa que esse sistema } \\
\text { de verificação e cuidados pode ser efetivo na } \\
\text { redução da PAV. }\end{array}$ \\
\hline $\begin{array}{l}\text { MYNY D, et al., } \\
2005\end{array}$ & $\begin{array}{l}\text { Pneumonia associada } \\
\text { ao ventilador em UTI de } \\
\text { cuidados terciários: análise de } \\
\text { fatores de risco para aquisição } \\
\text { e mortalidade. }\end{array}$ & $\begin{array}{l}1295 \\
\text { pacientes } \\
\text { incluídos }\end{array}$ & $\begin{array}{c}\text { A incidência de PAV em pacientes } \\
\text { na UTI ventilados por pelo menos } 48 \text { horas foi } \\
\text { de } 23,1 \% \text {. O tempo médio para o } \\
\text { desenvolvimento da PAV foi de } 9,6 \text { dias, com } \\
\text { mediana de } 6 \text { dia. }\end{array}$ & $\begin{array}{l}\text { Os pacientes com PAV tiveram } \\
\text { uma permanência significativamente mais longa } \\
\text { na UTI, com maior dependência da ventilação } \\
\text { mecânica e alta taxa de mortalidade. }\end{array}$ \\
\hline $\begin{array}{l}\text { FERREIRA CR, et } \\
\text { al., } 2016\end{array}$ & $\begin{array}{l}\text { A eficácia de um pacote na } \\
\text { prevenção de pneumonia } \\
\text { associada ao ventilador. }\end{array}$ & $\begin{array}{l}188 \text { pacientes } \\
\text { foram } \\
\text { incluídos. }\end{array}$ & $\begin{array}{l}\text { O pacote incluía } 10 \text { itens: cuidados com } \\
\text { alimentação, analgesia, sedação, profilaxia } \\
\text { tromboembólica, elevação da cabeceira do leito, } \\
\text { profilaxia da úlcera por estresse, controle } \\
\text { glicêmico, higiene bucal com clorexidina a } 2 \% \text {, } \\
\text { monitorização da pressão do manguito entre } 20 \\
\text { e } 25 \mathrm{~cm} \text { de água e sucção de secreções. }\end{array}$ & $\begin{array}{c}\text { Houve uma diminuição observável na ocorrência } \\
\text { de pneumonia associada ao ventilador nos } \\
\text { pacientes que faziam parte do pacote }(p<0,01) \text {, } \\
\text { bem como uma redução nas taxas de mortalidade } \\
(p<0,01) \text { e diminuição nos custos } \\
\text { hospitalares }(p<0,05) \text {. }\end{array}$ \\
\hline $\begin{array}{l}\text { PATTANSHETTY } \\
\text { RB, et al., } 2010\end{array}$ & $\begin{array}{l}\text { Efeito da fisioterapia } \\
\text { respiratória multimodal na } \\
\text { prevenção } \\
\text { de pneumonia associada } \\
\text { à ventilação mecânica: um } \\
\text { ensaio clínico randomizado }\end{array}$ & $\begin{array}{l}\text { Um total de } \\
101 \text { pacientes } \\
\text { foram } \\
\text { incluídos e } \\
\text { randomizados }\end{array}$ & $\begin{array}{c}\text { O grupo que recebeu intervenções } \\
\text { fisioterapêuticas como vibrações de } \\
\text { posicionamento na parede torácica, } \\
\text { Hiperinsuflação manual, aspiração traqueal e } \\
\text { fisioterapia respiratória obteve melhores } \\
\text { resultados. }\end{array}$ & $\begin{array}{l}\text { Observou-se melhores resultados no grupo de } \\
\text { estudo em comparação ao grupo controle que só } \\
\text { recebeu cuidados de rotina. A fisioterapia } \\
\text { respiratória duas vezes ao dia, esteve associada a } \\
\text { uma diminuição significativa de PAV e redução } \\
\text { significativa nas taxas de mortalidade. }\end{array}$ \\
\hline
\end{tabular}

REAS/EJCH | Vol.12(10) | e4306 | DOI: https://doi.org/10.25248/reas.e4306.2020 Página 6 de 10 


\begin{tabular}{|c|c|c|c|c|}
\hline SU M, et al., 2020 & $\begin{array}{l}\text { Probióticos para } \\
\text { a prevenção de pneumonia } \\
\text { associada ao ventilador: uma } \\
\text { meta-análise de ensaios } \\
\text { clínicos randomizados }\end{array}$ & $\begin{array}{l}\text { Quatorze } \\
\text { estudos que } \\
\text { envolveram } \\
1.975 \\
\text { indivíduos }\end{array}$ & $\begin{array}{l}\text { A administração de probióticos foi associada a } \\
\text { uma redução na incidência de PAV, o que } \\
\text { possibilitou uma redução do uso de antibióticos. }\end{array}$ & $\begin{array}{l}\text { Os resultados indicaram que a administração de } \\
\text { probióticos reduzem significativamente a incidência } \\
\text { de PAV e se configura como um tratamento eficaz } \\
\text { para pacientes já diagnosticados. }\end{array}$ \\
\hline $\begin{array}{l}\text { CARRASCOSA } \\
\text { DP, et al., } 2020\end{array}$ & $\begin{array}{l}\text { Drenagem de secreção } \\
\text { subglótica para prevenção de } \\
\text { pneumonia associada ao } \\
\text { ventilador: uma visão geral de } \\
\text { revisões sistemáticas e uma } \\
\text { meta-análise atualizada. }\end{array}$ & $\begin{array}{l}\text { A meta-análise } \\
\text { incluiu } 3684 \\
\text { adultos em } \\
\text { ventilação } \\
\text { mecânica e } \\
\text { internados na } \\
\text { UTI }\end{array}$ & $\begin{array}{l}\text { A drenagem de secreção subglótica reduziu } \\
\text { significativamente a incidência de PAV. }\end{array}$ & $\begin{array}{c}\text { As evidências sobre o uso da drenagem de } \\
\text { secreção subglótica para impedir a PAV, se } \\
\text { mostraram altas, houve ainda redução da } \\
\text { mortalidade. No entanto, não foram observados } \\
\text { impactos em outros desfechos, como diminuição da } \\
\text { ventilação mecânica na UTI / ou tempo de } \\
\text { internação hospitalar }\end{array}$ \\
\hline $\begin{array}{l}\text { ALEIXOU VG, et } \\
\text { al., } 2009\end{array}$ & $\begin{array}{l}\text { Impacto da posição do } \\
\text { paciente na incidência de } \\
\text { pneumonia associada ao } \\
\text { ventilador: uma meta-análise } \\
\text { de ensaios clínicos }\end{array}$ & $\begin{array}{l}\text { Total de } \\
\text { pacientes } \\
\text { incluídos: (337 } \\
\text { a 1.018) }\end{array}$ & $\begin{array}{l}\text { As chances de desenvolver PAV foram } \\
\text { significativamente menores entre } \\
\text { os pacientes encontrados na posição semi- } \\
\text { recumbente a } 45 \text { graus em comparação } \\
\text { aos pacientes na posição supina. }\end{array}$ & $\begin{array}{l}\text { A posição semirecumbentemente a } 45 \text { graus é a } \\
\text { mais indicada para a prevenção de PAV. }\end{array}$ \\
\hline $\begin{array}{l}\text { CHOUHDARI A, } \\
\text { et al., } 2018\end{array}$ & $\begin{array}{l}\text { Existe uma baixa taxa de } \\
\text { incidência de pneumonia } \\
\text { associada à ventilação } \\
\text { associada a menor } \\
\text { mortalidade? um estudo } \\
\text { longitudinal descritivo no Irã. }\end{array}$ & $\begin{array}{l}\text { Foram } \\
\text { avaliados } \\
1221 \\
\text { pacientes } \\
\text { internados na } \\
\text { UTI. }\end{array}$ & $\begin{array}{l}100 \text { pacientes ( } 8 \% \text { ) do total de } 1.221 \text { pacientes } \\
\text { analisados preencheram os critérios de } \\
\text { diagnóstico de PAV durante } 30 \text { dias. } \\
\text { Os indicadores para o surgimento de PAV } \\
\text { foram: Idade superior a } 40 \text { anos, mais de } 96 \\
\text { horas de VM e diabetes mellitus não controlado }\end{array}$ & $\begin{array}{c}\text { A taxa de mortalidade atribuível à PAV foi alta em } \\
\text { comparação com a taxa de mortalidade padrão. A } \\
\text { taxa de mortalidade mais alta foi ocasionada pelo } \\
\text { microorganismo Acinetobacter }\end{array}$ \\
\hline $\begin{array}{l}\text { ZENG H, et al., } \\
\qquad 2017\end{array}$ & $\begin{array}{l}\text { Efeito da fisioterapia } \\
\text { respiratória em pacientes } \\
\text { submetidos à ventilação } \\
\text { mecânica: um estudo } \\
\text { prospectivo randomizado } \\
\text { controlado. }\end{array}$ & $\begin{array}{l}\text { Foram } \\
\text { inscritos e } \\
\text { randomizados } \\
68 \text { pacientes } \\
\text { adultos } \\
\text { submetidos à } \\
\text { VM. }\end{array}$ & $\begin{array}{l}\text { O grupo controle recebeu fisioterapia de } \\
\text { rotina; enquanto o grupo intervenção recebeu } \\
\text { insuflação pulmonar manual, expectoração de } \\
\text { vibrações e exercício funcional precoce. }\end{array}$ & $\begin{array}{l}\text { A incidência de PAV no grupo intervenção foi } \\
\text { significativamente menor } 5,4 \% \text { em comparação ao } \\
\text { grupo controle } 25,8 \% \text {. Os pacientes no grupo } \\
\text { controle também apresentaram atelectasia, } \\
\text { trombose venosa profunda e outras complicações, } \\
\text { que não foram encontradas no grupo intervenção. }\end{array}$ \\
\hline
\end{tabular}

Fonte: FURTADO MVC, et al., 2020

REAS/EJCH | Vol.12(10) | e4306 | DOI: https://doi.org/10.25248/reas.e4306.2020 Página 7 de 10 


\section{DISCUSSÃO}

O estudo de Liu W, et al. (2019), descreve que o trato respiratório é um canal de troca entre as áreas interna e externa do corpo humano, o que também o torna o melhor caminho para que bactérias patogênicas de fora invadam o corpo humano. Quanto maior o tempo de uso do ventilador, mais bactérias crescerão e formarão biofilme no cateter traqueal. Neste segmento, bactérias mais patogênicas que crescem em um cateter levam a um risco maior de culminar em pneumonia associada a ventilação mecânica (PAV) e a chance de infecção será maior quanto mais duradouro for o tempo de uso da ventilação mecânica (VM). Os achados de Myny D, et al. (2005), seguem a mesma linha, demonstrando que a incidência de pneumonia associada a ventilação mecânica (PAV) entre os pacientes internados na unidade de terapia intensiva (UTI) é cerca de 23,1\% por pelo menos 48 horas de ventilação mecânica (VM) e que os pacientes com pneumonia associada a ventilação mecânica (PAV) possuem uma permanência mais longa na unidade de terapia intensiva (UTI).

Ainda no estudo de Liu W, et al. (2019), assim como no de Hua F, et al. (2016), há a orientação de que é importante para a proteção das vias aéreas os cuidados rigorosos de higiene bucal prestados aos pacientes que recebem ventilação mecânica (VM), pois até certo ponto, um estabelecimento artificial na via aéreas rompe a função de proteção natural que a cavidade oral e nasal possui contra bactérias patogênicas, o que possibilita o aumento da incidência de pneumonia associada a ventilação mecânica (PAV).

Com isso, foi verificado que a higienização bucal é uma conduta eficaz na prevenção de penumonia associada a ventilação (PAV), pois análises mostraram que cuidados como limpeza com enxaguatório bucal, cloredixina ou gel está associado a uma redução de $24 \%$ para cerca de $18 \%$ nas chances do paciente adquirir pneumonia associada a ventilação mecânica (PAV). Porém, as evidências são de baixa qualidade em relação ao impacto da higienização bucal na redução do tempo de ventilação mecânica (VM), diminuição da permanência na unidade de terapia intensiva (UTI) e redução da mortalidade.

Nesse sentido, outro tratamento eficaz foi a fisioterapia respiratória, observado por Zeng H, et al. (2017), onde as condutas fisioterapêuticas analisadas foram: insuflação pulmonar manual, expectoração de vibrações e exercícios funcionais, que obtiveram efeitos nos seguintes parâmetros: Escore fisiológico agudo, sistema de classificação de gravidade de doença (APACHE II), índice de relação entre a pressão arterial de oxigênio e a fração inspirada de oxigênio $\left(\mathrm{PaO}_{2} / \mathrm{FiO}_{2}\right)$ antes e após o tratamento, função respiratória e monitorização dos sinais vitais antes e após as condutas, o que se pode constatar pelas variáveis observadas que após as intervenções, o grupo que recebeu as condutas apresentou menor duração da ventilação mecânica (VM), o índice de relação entre a pressão arterial de oxigênio e a fração inspirada de oxigênio $\left(\mathrm{PaO}_{2} / \mathrm{FiO}_{2}\right)$ aumentou consideravelmente em 3 dias após o tratamento em comparação com o antes do tratamento, a saturação periférica de oxigênio $\left(\mathrm{SpO}_{2}\right)$ foi significativamente maior que antes do tratamento e o escore de sistema de classificação de gravidade de doença (APACHE II) diminuiu gradualmente.

Além do mais, sobre as medidas de tratamento Ferreira CR, et al. (2016), salienta sobre o uso de antibióticos, que são descritos na literatura científica como responsáveis por até $50 \%$ de todas as prescrições nas unidades de terapia intensiva (UTI) para o tratamento de pacientes com pneumonia associada a ventilação mecânica (PAV), porém, concluiu que dentre os vários fatores de risco para o aumento da incidência de pneumonia associada a ventilação mecânica (PAV) encontram-se o uso de mais de três tipos de antibióticos.

À vista disso, uma medida proposta por Su M, et al. (2020) e Johnstone J, et al. (2019), para reduzir a administração de antibióticos, foi realizar a administração de probióticos, que influenciam na microbiota, melhorando a função da barreira intestinal e reduzindo a carga bacteriana patogênica nos pacientes sobre cuidados intensivos, pois análises poderam mostrar que houve reduções significativas na incidência de pneumonia associada a ventilação mecânica (PAV) nos pacientes que fizeram uso de probióticos, o que possibilitou em uma duração mais curta na administração de antibióticos. Todavia, os autores não puderam mensurar o impacto dos probióticos em outros desfechos, como redução no índice de mortalidade, permanência na unidade de terapia intensiva (UTI) e diminuição dos dias em ventilação mecânica. 
No estudo realizado por Chouhdari $A$, et al. (2018), verificou-se que a taxa de incidência de pneumonia associada a ventilação mecânica (PAV) pode ser reduzida se medidas de prevenção forem adotadas, tais como: diminuição da duração da ventilação mecânica (VM), prevenção de intubação, diminuição da secreção do tubo endotraqueal e das cavidades orais e nasais através da aspiração, minimização da sedação, melhoramento do condicionamento físico e manutenção do circuito do ventilador.

Sendo o estudo de Carrascosa DP, et al. (2020), vindo também a corroborar com a realização da drenagem de secreção, pois analisando 20 ensaios clínicos randomizados (ECR's) e várias metanálises pode concluir que tal conduta foi capaz de reduzir a incidência de pneumonia associada a ventilação mecânica (PAV)..

Paralelo a isso, analisando medidas preventivas, Ferreira CR, et al. (2016), verificou que: cuidados com alimentação, analgesia, sedação, profilaxia tromboembólica, elevação da cabeceira do leito, profilaxia da úlcera por estresse, controle glicêmico, higiene bucal com clorexidina a $2 \%$, monitorização da pressão do manguito entre 20 e $25 \mathrm{~cm}$ de água e sucção de secreções subglótica, poderam promover redução da incidência de pneumonia associada a ventilação mecânica (PAV), redução dos custos hospitalares e nos índices de mortalidade, enfatizando-se que o rápido diagnóstico é fundamental para uma intervenção mais precoce e precisa, o que impacta diretamente no desfecho clínico mais positivo.

O autor salienta que para a detecção da pneumonia associada a ventilação mecânica (PAV) se deve verificar ao menos dois sinais clínicos e / ou anormalidades laboratoriais que sugerem um processo infeccioso, como: febre $\left(>38^{\circ} \mathrm{C}\right)$, leucocitose $\left(>10.000 \mathrm{~mm}^{-3}\right)$ ou leucopenia $\left(<4000 \mathrm{~mm}^{-3}\right)$ e presença de secreção traqueal purulenta após 48 horas de ventilação mecânica (VM).

De forma complementar, Aleixou VG, et al. (2009) e Wang L, et al. (2016), explicam que as medidas de prevenção e tratamento vão além das citadas anteriormente, demonstrando em seus estudos que o posicionamento do paciente no leito possui grande influência sobre a incidência de pneumonia associada a ventilação mecânica (PAV), pois observando os pacientes que estavam em posição supina, verificaram que o refluxo das secreções gástricas era maior, o que culminava em aspirações para o trato respiratório, causando assim um fator de risco para infecção pulmonar. Em contra partida, verificaram que os pacientes que estavam alocados na posição semi-reclinada entre $30^{\circ}$ a $60^{\circ}$ graus poderam ter reduções de refluxo do conteúdo gástrico, assim minimizando a aspiração desses liquidos para o trato respiratório.

Foram elucidados nos estudos de Myny D, et al. (2005) e Carrascosa DP, et al. (2020), que a pneumonia associada a ventilação mecânica (PAV) não foi tida exclusivamente como preditor independente de morte, tendo como alguns dos preditores um diagnóstico na admissão diferente de trauma, diabetes mellitus não controlada, idade superior a 40 anos e mais de 96 horas de ventilação mecânica (VM). No entanto, realizouse uma comparação entre pacientes com e sem pneumonia associada a ventilação mecânica (PAV) e se observou que os indivíduos com diagnóstico de pneumonia associada a ventilação mecânica (PAV), tinham taxas de mortalidade maiores $(22,2 \%)$ em relação aos sem pneumonia associada a ventilação mecânica (PAV) $(12,0 \%)$.

Chouhdari A, et al. (2018), corrobora com tais desfechos apresentando também em seu estudo uma taxa de mortalidade atribuível para pacientes afetados pela pneumonia associada a ventilação mecânica (PAV) bastante elevada, cerca de 34,8 , a $35,6 \%$, e concluiu que o tempo de sobrevida diminui com o aumento do tempo de permanência na unidade de terapia intensiva (UTI), sendo verificado também que o custo adicional da pneumonia associada a ventilação mecânica (PAV) é estimado em cerca de 700 US \$ por paciente.

Dessa forma, Ferreira CR, et al. (2016), explica que muitos casos de pneumonia associada a ventilação mecânica (PAV) podem ser evitados prestando muita atenção ao realizar a assistência, e que se deve preparar a equipe para as consultas, ajudando a identificar e prevenir erros de medicação, promovendo assim a segurança do paciente e maximizando intervenções terapêuticas dentro da unidade de terapia intensiva através do debate técnico entre os membros da equipe multiprofissional. 


\section{CONSIDERAÇÕES FINAIS}

Podemos concluir que a pneumonia associada a ventilação mecânica é uma infecção hospitalar que possui diversos fatores preditores para a sua ocorrência e que está relacionada a maior duração da ventilação mecânica, maior índice de mortalidade e maiores gastos hospitalares. As medidas como drenagem de secreção subglótica, hiperinsuflação manual, fisioterapia respiratória, exercícios precoces, administração de probiotocos, higienização bucal e monitorização frequente do paciente se mostraram eficientes na diminuição da incidência e no tratamento da pneumonia associada à ventilação mecânica. No entanto, as evidências sobre os impactos em outros desfechos como aumento da sobrevivência, diminuição dos dias em ventilação mecânica e diminuição da permanência na unidade de terapia intensiva, foram observados em poucos estudos, sugerindo assim novas pesquisas de longa escala e mais detalhadas para determinar se os tratamentos e medidas preventivas diminuem não só a incidência de pneumonia, mas também impactam em outros desfechos clínicos.

\section{AGRADECIMENTOS}

Nosso agradecimento à Liga Acadêmica de Fisioterapia em Terapia Intensiva do Estado do Pará (LAFITIPA), por nos estimular a seguir a prática baseada em evidências e à Escola Superior da Amazônia (ESAMAZ), por nos fornecer mecanismos técnicos e literários para o desenvolvimento de projetos de pesquisas.

\section{REFERÊNCIAS}

1. CHOUHDARI A, et al. Is a Low Incidence Rate of ventilation associated pneumonia associated whith lower mortality? a Descriptive Longitudinal Study in Iran. Tanaffos Journal. 2018; 17(2): 110-116

2. Agência Nacional de Vigilância Sanitária. 2017. in: Caderno 1: Assistência segura: uma reflexão teórica aplicada à prática. Disponível em: http://www20.anvisa.gov.br/segurancadopaciente Acesso em: 05 Mar. 2020

3. Agência Nacional de Vigilância Sanitária. 2017. in: Caderno 4: Medidas de Prevenção de Infecções Relacionadas à Assistência à Saúde. Disponível em: http://www20.anvisa.gov.br/segurancadopaciente. Acesso em: 05 Mar. 2020

4. CARVALHO CRR. Pneumonia associada à ventilação mecânica. Jornal brasileiro de pneumologia. 2006;32(4):20-22

5. FERREIRA CR, et al. The effectiveness of a bundle in the prevention of ventilator-associated pneumonia. The Brazilian Journal of Infectious Diseases. 2016; 20(3): 267-271

6. HUA F, et al. Oral hygiene care for critically ill patients to prevent ventilator-associated pneumonia. Cochrane Database Syst Rev. 2016; (10): CD008367

7. MYNY D, et al. Ventilator-associated pneumonia in a tertiary care ICU: analysis of risk factors for acquisition and mortality. International Journal of Clinical and Laboratory Medicine. 2005; 60 (3): 114-2

8. RODRIGUES AN, et al. Impactos e fatores determinantes no bundle de pneumonia associada à ventilação mecânica. Revista Brasileira de Enfermagem. 2016; (9):1984-0446

9. ALEIXOU VG, et al. Impact of patient position on the incidence of ventilator-associated pneumonia: A meta-analysis of randomized controlled trials. Journal of Critical Care. 2009; 24 (4): 515-22

10. SU M, et al. Probiotics for the Prevention of Ventilator-Associated Pneumonia. A Meta-Analysis Of Randomized Controlled Trials. Respiratory Care. 2020; (3): 07097

11. CARRASCOSA DP, et al. Subglotic secretion drainage for preventing ventilador-associated pneumonia: an overview of systematic reviews and an updated meta-analysis. Europen Respiratory Review. 2020; (20): 190107

12. LIU W, et al. Active surveillance of ventilator-associated pneumonia in the intensive care unit and establishment of the risk grading system and effect evaluation. Annals of Translational Medicine. 2019; 7(22): 617

13. PATTANSHETTY RB, et al. Effect of multimodality chest physiotherapy in prevention of ventilator-associated pneumonia: a randomized clinical trial. Indian Journal of Critical Care Medicine. 2010;14(2):70-76

14. ZENG H, et al. Effect of chest physiotherapy in patients undergoing mechanical ventilation: a prospective randomized controlled trial. Zhonghua Wei Zhong Bing Ji Jiu Yi Xue. 2017; 29(5):403-406

15. WANG L, et al. Semi-recumbent position versus supine position for the prevention of ventilator-associated pneumonia in adults requiring mechanical ventilation. Cochrane Database Syst Rev. 2016; (1): CD009946

16. JOHNSTONE J, et al. Evaluating probiotics for the prevention of ventilator-associated pneumonia: a randomised placebo-controlled multicentre trial protocol and statistical analysis plan for PROSPECT. BMJ open. 2019; 9(6): e025228

17. BARBAS CSV, et al. Recomendações brasileiras de ventilação mecânica parte I. Revista Brasileira de Terapia Intensiva. 2014; 26(2):89-121

18. YAMANAKA CS, et al. Intubação orotraqueal: avaliação do conhecimento medico e das práticas clínicas adotadas em unidades de terapia intensiva. Revista Brasileira de Terapia Intensiva. 2010; 22(2): 103-111

19. ROQUILLY A, et al. Pneumonia Prevention to Decrease Mortality in intensive Care Unit: A systematic Review And Meta-Analysis. Clinical Infectious Diaseases. 2015; 60(1): 64-75

20. BARROS $\mathrm{F}$, et al. Adhesión al bundle de prevención de neumonia asociada a la ventilación mecánica. Revista Cuidarte. 2020; 2346-341

21. HESPANHOL L, et al. Infección relacionada con la Asistencia a la Salud em Unidad de Cuidados Intensivos. Revista Enfermería Global. 2019; 1695-6141. 